\title{
Schlüter, Martin
}

\section{Zum Studium der Anthroposophie}

Demisch, Ernst-Christian [Hrsg.]; Kiersch, Johannes [Hrsg.]; Greshake-Ebding, Christa [Hrsg.]: Steiner neu lesen. Perspektiven für den Umgang mit Grundlagentexten der Waldorfpädagogik. Frankfurt, M. : Lang 2014, S. 21-35. (Kulturwissenschaftliche Beiträge der Alanus Hochschule für Kunst und Gesellschaft; 12)

Quellenangabe/ Reference:

Schlüter, Martin: Zum Studium der Anthroposophie - In: Demisch, Ernst-Christian [Hrsg.]; Kiersch, Johannes [Hrsg.]; Greshake-Ebding, Christa [Hrsg.]: Steiner neu lesen. Perspektiven für den Umgang mit Grundlagentexten der Waldorfpädagogik. Frankfurt, M. : Lang 2014, S. 21-35 - URN: urn:nbn:de:0111-pedocs-128777 - DOI: 10.25656/01:12877

https://nbn-resolving.org/urn:nbn:de:0111-pedocs-128777

https://doi.org/10.25656/01:12877

in Kooperation mit / in cooperation with:

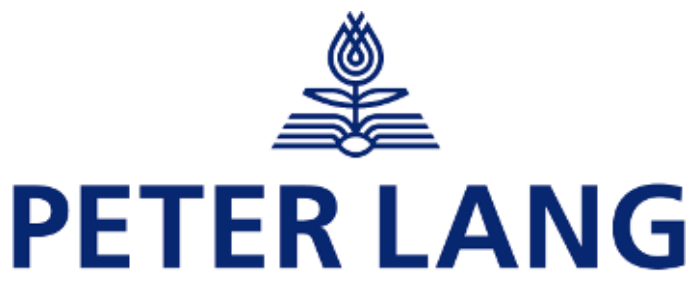

INTERNATIONALER VERLAG DER WISSENSCHAFTEN

http://www.peterlang.com

\section{Nutzungsbedingungen}

Gewährt wird ein nicht exklusives, nicht übertragbares, persönliches und beschränktes Recht auf Nutzung dieses Dokuments. Dieses Dokument ist ausschließlich für den persönlichen, nicht-kommerziellen Gebrauch bestimmt. Die Nutzung stellt keine Übertragung des Eigentumsrechts an diesem Dokument dar und gilt vorbehaltlich der folgenden Einschränkungen Auf sämtlichen Kopien dieses Dokuments müssen alle Urheberrechtshinweise und sonstigen Hinweise auf gesetzlichen Schutz beibehalten werden. Sie dürfen dieses Dokument nicht in irgendeiner Weise abändern, noch dürfen Sie dieses Dokument für öffentliche oder kommerzielle Zwecke vervielfältigen, öffentlich ausstellen, aufführen, vertreiben oder anderweitig nutzen.

Mit der Verwendung dieses Dokuments erkennen Sie die Nutzungsbedingungen an.

\section{Terms of use}

We grant a non-exclusive, non-transferable, individual and limited right to using this document.

This document is solely intended for your personal, non-commercial use. Use of this document does not include any transfer of property rights and it is conditional to the following limitations: All of the copies of this documents must retain all copyright information and other information regarding legal protection. You are not allowed to alter this document in any way, to copy it for public or commercial purposes, to exhibit the document in public, to perform, distribute or otherwise use the document in public.

By using this particular document, you accept the above-stated conditions of use.

\section{Kontakt / Contact:}

\section{peDOcs}

DIPF | Leibniz-Institut für Bildungsforschung und Bildungsinformation Informationszentrum (IZ) Bildung

E-Mail:pedocs@dipf.de

Internet: www.pedocs.de

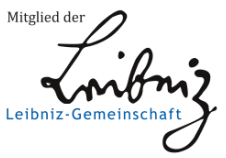




\section{Kulturwissenschaftliche Beiträge}

der Alanus Hochschule

für Kunst und Gesellschaft

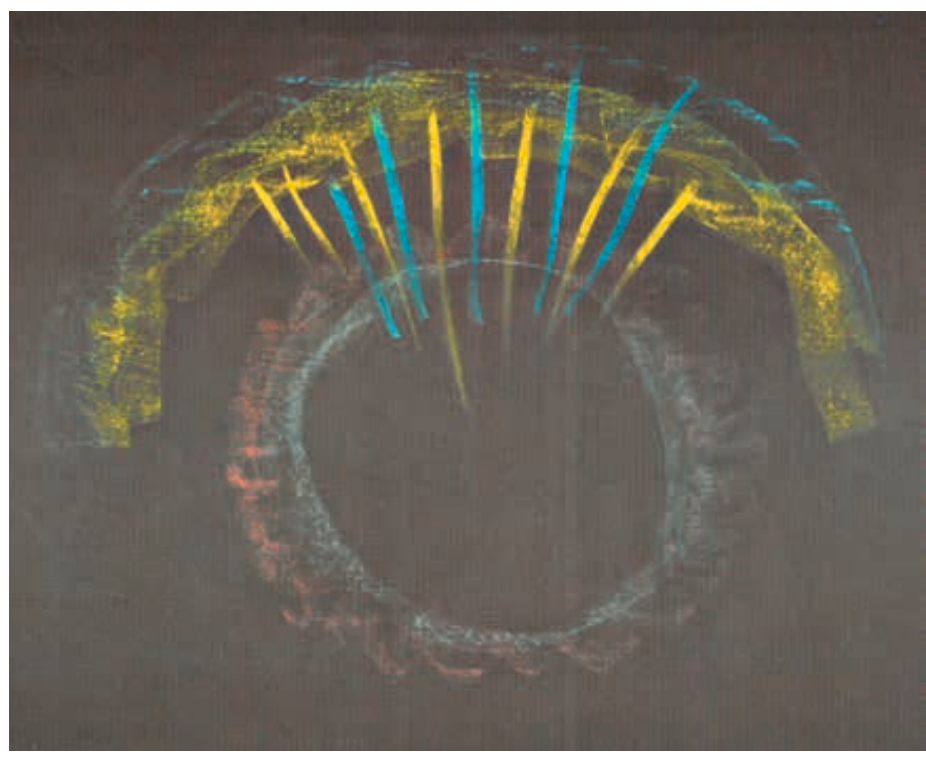

Ernst-Christian Demisch / Christa Greshake-Ebding / Johannes Kiersch / Martin Schlüter / Gerhard Stocker (Hrsg.)

Steiner neu lesen

Perspektiven für den Umgang mit Grundlagentexten der Waldorfpädagogik 
Die Anthroposophie Rudolf Steiners wird gegenwärtig neu erforscht, als historisches Phänomen ebenso wie als Impulsgeber für aktuelle Projekte in vielen Lebensfeldern. Dabei geht es auch um ein sachgemäßes Verstehen der zugrunde liegenden Quellentexte. Dieses Buch beleuchtet die dabei zu lösenden, bisher übersehenen hermeneutischen Probleme. Es diskutiert die besonderen Ausdrucksmittel, die Steiner in seinen Schriften und Vorträgen verwendet, revidiert die verbreitete Ansicht, dass es sich bei anthroposophischen Einsichten um ein Faktenwissen im Sinne empirischer Forschung der üblichen Art handle, und eröffnet damit Perspektiven für einen undogmatischen, offenen Umgang mit dem bis heute umstrittenen Werk des Pädagogen und Lebensreformers.

Die Herausgeber dieses Sammelbandes arbeiten im Institut für Waldorfpädagogik in Witten als Ausbilder für Lehrkräfte an Waldorfschulen. 
Steiner neu lesen 


\title{
Kulturwissenschaftliche Beiträge
}

der Alanus Hochschule für Kunst und Gesellschaft

\author{
Herausgegeben von der \\ Alanus Hochschule für Kunst und Gesellschaft \\ Peter Schneider und Marcelo da Veiga
}

Band 12 
Ernst-Christian Demisch / Christa Greshake-Ebding / Johannes Kiersch / Martin Schluiter / Gerhard Stocker (Hrsg.)

\section{Steiner neu lesen}

Perspektiven für den Umgang mit Grundlagentexten der Waldorfpädagogik 
Bibliografische Information der Deutschen Nationalbibliothek Die Deutsche Nationalbibliothek verzeichnet diese Publikation in der Deutschen Nationalbibliografie; detaillierte bibliografische Daten sind im Internet über http://dnb.d-nb.de abrufbar.

\author{
Umschlagabbildung: \\ Unter Verwendung eines Motivs aus \\ Wandtafelzeichnungen Rudolf Steiners
}

ISSN 1861-4531

ISBN 978-3-631-64969-5 (Print)

E-ISBN 978-3-653-04113-2 (E-Book)

DOI 10.3726/978-3-653-04113-2

(c) Peter Lang GmbH

Internationaler Verlag der Wissenschaften

Frankfurt am Main 2014

Alle Rechte vorbehalten.

Peter Lang Edition ist ein Imprint der Peter Lang GmbH.

Peter Lang - Frankfurt am Main · Bern · Bruxelles .

New York · Oxford - Warszawa $\cdot$ Wien

Das Werk einschließlich aller seiner Teile ist urheberrechtlich geschützt. Jede Verwertung außerhalb der engen Grenzen des

Urheberrechtsgesetzes ist ohne Zustimmung des Verlages unzulässig und strafbar. Das gilt insbesondere für Vervielfältigungen, Übersetzungen, Mikroverfilmungen und die Einspeicherung und Verarbeitung in elektronischen Systemen.

Dieses Buch erscheint in der Peter Lang Edition und wurde vor Erscheinen peer reviewed.

www.peterlang.com 


\title{
Zum Studium der Anthroposophie
}

\author{
Martin Schlüter
}

\begin{abstract}
„Das 19. Jahrhundert hat die Industrialisierung dessen erlebt, was menschliche Hände machen können. Das 20. Jahrhundert hat die Industrialisierung der menschlichen Fortbewegungsorgane vollzogen. Jetzt erleben wir die Industrialisierung des Gehirns."
\end{abstract}

Eine Flut von Eindrücken und Informationen strömt heute täglich auf uns ein. Mehr und mehr kann es nur vorsätzlich geschehen, das wir uns dieser Reizüberflutung entziehen, besonnen auf sie schauen, Wesentliches von Unwesentlichem unterscheiden und dem, was uns wirklich betrifft unsere ganze Aufmerksamkeit schenken. Bei diesem Vorgang wenden wir eine Kraft des Denkens an, die in der gewöhnlichen Alltagsbewältigung mit strömt, ohne dass wir sie sonderlich beachten: Beim Zurücktreten von den heranflutenden Eindrücken verwenden wir das Denken als ein Anschauungsorgan, das sich eines (vor-)schnellen Urteilen enthält, aber gerade dadurch das Wesentliche gewahr wird und so Orientierung schafft.

Johann Wolfgang v. Goethe und Rudolf Steiner haben beide auf verschiedenen Wegen eine Denkungsart entwickelt, deren Quelle diese Anschauungskraft ist. Eine Denkungsart, die nicht dabei stehenbleibt über die Dinge und Wesen nachzudenken, sondern die sie vielmehr in Gedankenform erstehen ${ }^{2}$ und so $\mathrm{ihr}$ Wesen unmittelbar erlebbar werden lässt. Goethe wandte sich dabei den Naturreichen zu und gestaltete seine , gegenständliche' Denkungsart ${ }^{3}$ zu einer naturwissenschaftlichen Methode aus ${ }^{4}$, die er für weit bedeutender hielt, als all sein dichterisches Schaffen. ${ }^{5}$ Rudolf Steiner war schon vor seiner Beschäftigung mit Goethe zu dieser Weltsicht gekommen ${ }^{6}$. Anders als Goethe begründete er sie aber philosophisch und entwickelte sie zu einem gedanklichen Erleben der übersinnlichen Welt weiter. Von der Erkenntnisentwicklung dieser beiden Persönlichkeiten soll im Folgenden zunächst die Rede sein.

1 Carr, N.: „Wer bin ich, wenn ich online bin und was macht mein Gehirn solange? Wie das Internet unser Denken verändert“, Blessing Verlag München 2010

2 Samuel, R. (Hrsg.): Novalis Schriften Bd.II, S. 640ff, Stuttgart 1981

3 Goethe, J.W.: Bedeutende Fördernis durch ein einziges geistreiches Wort, Hamburger Ausgabe, Bd. 13, S.37, München 1981

4 Goethe an Hegel am 7. Oktober 1820. In: Hamburger Ausgabe, Briefe Bd. III, S.492, München 1986

5 Kroeber, H.T. (Hrsg.): Goethe - Gespräche mit Eckermann, S.278. Weimar 1918

6 Steiner, R.: Grundlinien einer Erkenntnistheorie der Goetheschen Weltanschauung, GA 2, Vorrede 1923 und Beiträge zur Rudolf Steiner Gesamtausgabe, Nr.63., S.5ff. Dornach 1978 


\section{Die Entfaltung der Goetheschen Denkungsart}

Goethes Verhältnis zur Natur hat im Laufe seines Lebens Verwandlungen durchgemacht. Ein Grundzug erwies sich jedoch als eine verlässliche Konstante, die durch seine Begegnung mit Schiller hervortrat und deutlichere Konturen erhielt.

Blicken wir zunächst in die Kindheit Goethes, so finden wir den etwa achtjährigen Knaben, wie er den Gesprächen seiner Eltern und deren Freunden über ,den rechten Glauben' folgt. Angeregt durch diese Auseinandersetzungen

„... kam er auf den Gedanken, sich dem großen Gotte der Natur, dem Schöpfer und Erhalter Himmel und der Erden ... unmittelbar zu nähern ... Der Gott, der mit der Natur in unmittelbarer Verbindung stehe, sie als sein Werk anerkenne und liebe, dieser schien ihm der eigentliche Gott ... Eine Gestalt konnte der Knabe diesem Wesen nicht verleihen; er suchte ihn also in seinen Werken auf, und wollte ihm auf gut alttestamentliche Weise einen Altar errichten. Naturprodukte sollten die Welt im Gleichnis vorstellen, über diesen sollte eine Flamme brennen und das zu seinem Schöpfer sich aufsehnende Gemüt des Menschen bedeuten. “7

So suchte er aus der Naturaliensammlung die besten Stücke heraus und drapierte sie auf einem mehrstufigen, pyramidenförmigen, rot lackierten und goldgeblümten Notenpult, ,so dass es heiter und zugleich bedeutend genug aussah. " Ein Räucherkerzchen wurde in einer Porzellanschale auf die abgeflachte Spitze gestellt, jedoch nicht einfach entzündet: der Knabe wartete ab, bis die Sonne über den Frankfurter Dächern so weit aufgestiegen war, dass er es mit Hilfe eines Brennglases zum Glimmen bringen konnte. ,Alles gelang nach Wunsch und die Andacht war vollkommen. “ Der Knabe ,, sehnte sich nach Wiederholung der Feierlichkeit", unglücklicherweise war aber zur gelegenen Zeit die Porzellantasse nicht zur Hand , und die Andacht war so groß, dass der Priester nicht merkte, welchen Schaden sein Opfer anrichtete, als bis ihm nicht mehr abzuhelfen war. " Der Mut zu neuen Opfern war ihm vergangen...

Im religiösen Gemüt Goethes lebte von Kindheit an die Empfindung, dass der Schöpfergott eins mit seinem Werke sei. Geist und Natur sind für ihn untrennbar verbunden. Eine künstlerische Form erhält diese Grundempfindung etwa ein Vierteljahrhundert später in einem aphoristischen Fragment über die Natur:

„Natur! Wir sind von ihr umgeben und umschlungen - unvermögend, aus ihr herauszutreten und unvermögend tiefer in sie hinein zu kommen ... gedacht hat sie und sinnt beständig, aber nicht als Mensch, sondern als Natur." ${ }^{\text {8 }}$

7 Goethe, J.W.: Dichtung und Wahrheit, 1. Buch. Hamburger Ausgabe, Band 9, S. 43ff., München 1982

8 Goethe, J.W.: Die Natur (Fragment), Hamburger Ausgabe Bd.13, S. 45 ff., München 1982 
Die Natur ,denkt' in ihren Verwandlungen. Das Gesetz, die Notwendigkeit ist unmittelbar mit den Erscheinungen verbunden, ist eins mit ihnen. Der erkennende Mensch hat daher die Aufgabe, sich dieses im Naturzusammenhang wirkende ,Denken der Natur' in Gestalt von Ideen bewusst zu machen. Das versuchte Goethe seit Ende der siebziger Jahre des achtzehnten Jahrhunderts besonders hinsichtlich der Pflanzenwelt: Woher wissen wir, auch bei unbekannten Formen, dass es sich bei einer bestimmten Gestalt um eine Pflanze handelt? Es muss ein Urbild, eine Urpflanze geben, die der Möglichkeit nach alle Pflanzen in sich enthält. Goethe suchte diese Urpflanze im Zusammenhang aller bekannten und von Linné klassifizierten Arten und meinte sie auf seiner italienischen Reise gefunden zu haben. So schrieb er 1787 an seine Freundin Charlotte von Stein nach Weimar:

„Sage Herdern, dass ich dem Geheimnis der Pflanzenzeugung und Organisation ganz nahe bin und dass es das einfachste ist, was nur gedacht werden kann ... Sage ihm, dass ich den Hauptpunkt, wo der Keim stickt ganz klar und zweifellos entdeckt habe, dass ich alles übrige auch schon im Ganzen übersehe und nur noch einige Punkte bestimmter werden müssen. Die Urpflanze wird das wunderlichste Geschöpf von der Welt über welches mich die Natur selbst beneiden soll. Mit diesem Modell und dem Schlüssel dazu, kann man alsdann Pflanzen ins Unendliche erfinden, die konsequent sein müssen, das heißt: die, wenn sie auch nicht existieren, doch existieren könnten und nicht etwa malerische oder dichterische Schatten und Scheine sind, sondern innerliche Wahrheit und Notwendigkeit haben." ${ }^{\text {(9) }}$

\section{Der moderne Mensch: Schiller}

Ganz anders Schiller: In einem dritten Versuch legte der gerade 21-jährige Schiller dem Prüfungsausschuss der hohen Karlsschule in Stuttgart eine medizinische Dissertation vor mit dem Titel „Über den Zusammenhang der tierischen Natur des Menschen mit seiner geistigen "10. Natur und Geist sind für Schiller wie für uns heute auch - zwei völlig verschiedene, ja sich fremde Seiten der Wirklichkeit. Sie üben jeweils - durch die Vernunft einerseits und die Triebe auf der anderen - ihren unausweichlichen Einfluss auf den Menschen aus. Die Frage, ob der Mensch unter diesen Einflüssen ein freies Wesen sei oder werden könne, durchzieht das ganze philosophische Streben Schillers. Er suchte sie zunächst in der Aufmerksamkeit, in Liebe und Schönheit, in Anmut und Würde und schließlich in Steigerung und spielerischem Ausgleich der polaren Kräfte. Goethe hätte die Lebensfrage Schillers nach dem Zusammenhang von Geist und

9 Goethe an Charlotte v. Stein am 8. Juni 1787. In: Hamburger Ausgabe Briefe, Bd. II, S.60, München 1986

10 Schiller, F.: Sämtliche Werke, Bd. V., S. 287 ff. Darmstadt 1993. 
Stoff, von Idee und Sinneserfahrung nie stellen können, bildete dieser doch als selbstverständlich gegeben, und nur zu erhellender, den Ausgangs- und Zielpunkt seines naturwissenschaftlichen Strebens. So ist es nicht verwunderlich, dass Goethe besonders nach seiner Rückkehr aus Italien eine wirkliche Begegnung mit Schiller vermied, obgleich dieser sie suchte und Freunde wiederholt Gelegenheiten arrangierten.

\section{Die Begegnung}

Das erste wirkliche Gespräch zwischen ihnen ergab sich dann eher zufällig, als beide gleichzeitig am Sonntag, den 22. Juli 1794 aus einem naturwissenschaftlichen Vortrag in Jena kamen: Man tauschte Höflichkeiten aus, woraus sich ein Gespräch entwickelte, von welchem Goethe folgendermaßen berichtete:

„....er [Schiller, d.V.] schien an dem Vorgetragenen teilzunehmen, bemerkte aber sehr verständig und einsichtig und mir sehr willkommen, wie eine so zerstückelte Art die Natur zu behandeln, den Laien, der sich gerne darauf einließe, keineswegs anmuten könne.

Ich erwiderte darauf: dass sie dem Eingeweihten selbst vielleicht unheimlich bleibe, und dass es doch wohl noch andere Weisen geben könne, die Natur nicht gesondert und vereinzelt vorzunehmen, sondern sie wirkend und lebendig, aus dem Ganzen in die Teile strebend darzustellen. Er wünschte darüber aufgeklärt zu sein, verbarg aber seine Zweifel nicht..." “1l

Sie kamen zu seinem Haus, Goethe ging mit hinein (!) und trug Schiller lebhaft die Metamorphose der Pflanzen vor und ,ließ mit wenigen charakteristischen Federstrichen, eine symbolische Pflanze vor seinen Augen entstehen." Schiller war seinen Ausführungen aufmerksam und mit entschiedener Fassungskraft gefolgt, schüttelte aber den Kopf und sagte: „Das ist keine Erfahrung, das ist eine Idee. "Worauf Goethe einigermaßen zornig sagte: „Das kann mir sehr lieb sein, dass ich Ideen habe ohne es zu wissen und sie sogar mit Augen sehe. "Mit dieser kurzen Bemerkung machte Schiller, ohne es zu ahnen, auf eine Qualität von Goethes Denken aufmerksam, die vor ihm nur im alten Griechenland zu finden war, dort aber als gewöhnliches Erleben aller Menschen. So schrieb Aristoteles: „,Die Tätigkeit des Geistes ist das Denken, nämlich das Anschauen der Denkgegenstände; so wie die Tätigkeit des Gesichtsorgans das Sehen des Sichtbaren ist. " 12 Goethe hatte diese in Griechenland allgemein erlebte Qualität des Denkens, gleichsam dessen objektive Seite durch die Art seines Erkenntnisstrebens,

11 Goethe, J.W.: Glückliches Ereignis. In: Hamburger Ausgabe, Bd.10, S. 538 ff., München 1982

12 Aristoteles, Protreptikos. Zitiert nach Dietz, K.-M.: Metamorphosen des Geistes, Bd. II, S. 11. Stuttgart 1989. 
wie wir es zu skizzieren versuchten, gepflegt und ausgebildet, ohne sich der Besonderheit gegenüber seinen Zeitgenossen klar bewusst zu sein. Erst durch Schiller wurde er auf die angelegte und individuell ausgebildete Anschauungsraft seines Denkens aufmerksam.

Die ersten Briefe, welche die beiden wenige Wochen später austauschten, lassen das deutlich hervortreten. Etwa einen Monat nach ihrer ersten wirklichen Begegnung, am 23. August 1794 schrieb Schiller:

„... Lange schon habe ich, obgleich aus ziemlicher Ferne dem Gang ihres Geistes zugesehen und den Weg, den sie sich vorgezeichnet haben, mit immer erneuerter Bewunderung bemerkt. Sie suchen das Notwendige in der Natur, aber sie suchen es auf dem schweresten Wege, vor welchem jede schwächere Kraft sich wohl hüten würde. Sie nehmen die ganze Natur zusammen, um über das Einzelne Licht zu bekommen; in der Allheit der Erscheinungsarten suchen Sie den Erklärungsgrund für das Individuum auf ... Wären Sie als ein Grieche ... geboren worden und hätte schon von der Wiege an eine auserlesene Natur und eine idealisierende Kunst Sie umgeben, so wäre ihr Weg unendlich verkürzt, vielleicht ganz überflüssig gemacht worden ... Nun, da Sie als ein Deutscher geboren sind, da Ihr griechischer Geist in diese nordische Schöpfung geworfen wurde, so blieb Ihnen keine andere Wahl, als ... gleichsam von innen heraus und auf rationalem Wege ein Griechenland zu gebären. "13

Goethe antwortete nur wenige Tage später:

„Zu meinem Geburtstage, der mir diese Woche erscheint, hätte mir kein angenehmer Geschenk werden können als Ihr Brief, in welchem Sie, mit freundschaftlicher Hand, die Summe meiner Existenz ziehen ..." “14

In der anschließenden Zusammenarbeit Goethes und Schillers - methodisch vor allem in der Farbenlehre - wurde Goethe die Eigenart seines Denkens, deren Besonderheit auch Novalis beschrieb ${ }^{15}$, immer deutlicher bewusst, so dass er sie gezielter pflegen und ausbilden konnte. Eine große Freude war ihm folgende Würdigung Heinroths lange nach Schillers Tod:

„Herr Dr. Heinroth in seiner Anthropologie ... spricht von meinem Wesen und Wirken günstig, ja er bezeichnet meine Verfahrensweise als eine eigentümliche: dass nämlich mein Denken gegenständlich tätig sei, womit er aussprechen will, dass mein Denken sich von den Gegenständen nicht sondere, dass die Elemente der Gegenstände, die Anschauungen in dasselbe eingehen und von ihm durchdrungen werden, dass mein Anschauen selbst ein Denken und mein Denken ein Anschauen sei; welchem Verfahren genannter Freund seinen Beifall nicht versagen will.“ 16

13 Schiller an Goethe, 23. August 1794. In: Briefwechsel, S. 10f.. Fischer Verlag, Frankfurt 1961.

14 a.a.O., S. $12 \mathrm{f}$..

15 Siehe Anm. 2

16 Siehe Anm. 3 
Goethe hatte in seiner naturwissenschaftlichen Arbeit das Denken selbst zu einem Anschauungsorgan entwickelt. Dies versuchte auch C. F. v. Weizsäcker zu beschreiben, wenn er sagt:

„Goethe sah die Urpflanze wirklich. Es ist schon ein Ausweichen in einen Dualismus, wenn wir sagen, er habe sie mit dem inneren Auge gesehen. Lieber würde ich sagen, er sah sie mit dem denkenden Auge; er sah sie mit seinen leibhaften Augen, weil er denkend zu sehen vermochte. “17

\section{Rudolf Steiners Weg}

In jenem Alter etwa, in welchem Goethe als ,junger Priester' seinem Gott ein Opfer brachte, hatte Rudolf Steiner ein wichtiges übersinnliches Erlebnis:

„Die Schwester meiner Mutter war auf tragische Art gestorben. Der Ort an dem sie lebte, war ziemlich weit von dem unsrigen entfernt. Meine Eltern hatten keine Nachricht. Ich sah, sitzend im Wartesaal des Bahnhofs das ganze Ereignis. Ich machte einige Andeutungen in Gegenwart meines Vaters und meiner Mutter. Sie sagten nur: ,Du bist a dummer Bua'. In einigen Tagen sah ich, wie mein Vater nachdenklich wurde durch einen erhaltenen Brief, wie er dann, ohne mein Beisein nach einigen Tagen mit meiner Mutter sprach und diese dann tagelang weinte. Von dem tragischen Ereignisse erfuhr ich erst nach Jahren... Von jenem Ereignisse ab, fing für den Knaben ein Leben in der Seele an, in welchem sich durchaus die Welten offenbaren, aus denen nicht nur die äußeren Bäume, die äußeren Berge zu der Seele des Menschen sprechen, sondern auch jene Welten, die hinter dieser sind. Und der Knabe lebte etwa von jenem Zeitpunkte an, mit den Geistern der Natur, die ja in einer solchen Gegend besonders zu beobachten sind." ${ }^{\prime 18}$

Ein ganzes Menschenleben nach diesem Erlebnis machte Rudolf Steiner in einem Vortrag darauf aufmerksam, dass das Erleben der geistigen Welt, wie es damals für ihn begonnen hatte, niemals ,irgendwie abgerissen' war:

„Es war immer da. Ich muss das erwähnen, weil das den Hintergrund dieser Betrachtungen bilden muss: die geistige Welt eine Selbstverständlichkeit, und die Menschen auf Erden eben betrachtet als die Abbilder desjenigen, was sie eigentlich als geistige Wesenheiten innerhalb der geistigen Welt sind. Diese Gemütsverfassung möchte ich eben charakterisieren, damit sie dieses als geistigen Hintergrund immer voraussetzen.“19

17 Weizsäcker, C. F. v.: Goethes Naturwissenschaft. Hamburger Ausgabe, Bd. 13, S. 544. München 1982

18 Steiner, R.: Autobiographischer Vortrag über Kindheits- und Jugendjahre vom 4.2.1913. In: Beiträge zur Gesamtausgabe Nr. 83/84, Seite 2.

19 Steiner, R.: Die Geschichte und die Bedingungen der anthroposophischen Bewegung, GA 258, Vortrag vom 10.6.1923, S. 11ff.. Dornach 1981 
Jugendfreundschaften erlebte Rudolf Steiner daher auf besondere Weise, sie

„...hatten zum Fortgang meines Lebens ein eigentümliches Verhältnis. Sie zwangen mich zu einer Art Doppelleben in der Seele. Das Ringen mit den Erkenntnisrätseln, das vor allem damals meine Seele erfüllte, fand bei meinen Freunden zwar stets starkes Interesse, aber wenig mit tätigen Anteil. Ich blieb im Erleben dieser Rätsel ziemlich einsam. Dagegen lebte ich selbst alles voll mit, was im Dasein meiner Freunde auftauchte. So gingen zwei Lebensströmungen in mir nebeneinander: eine, die ich wie ein einsamer Wanderer verfolgte, und die andere, die ich in lebendiger Geselligkeit mit liebgewonnenen Menschen durchmachte. “20

Seit früher Kindheit lebte Rudolf Steiner nicht nur mit den Wesen der Natur, ,die man sieht', sondern auch mit der geistigen Welt, mit Wesen, ,die man nicht sieht'. Aber es fand sich zunächst kein Mittel der Übersetzung, des Sprechens von seinen Erlebnissen und den sich daraus ergebenden Fragen. Erst mit etwa neun Jahren entdeckte er zu seiner großen Freude einen Bereich rein geistigen Erlebens, den alle Menschen teilen können:

„Bald nach meinem Eintritt in die Neudörfler Schule entdeckte ich in seinem Zimmer ein Geometriebuch. Ich stand so gut mit diesem Lehrer, dass ich das Buch ohne weiteres eine Weile zu meiner Benutzung haben konnte. Mit Enthusiasmus machte ich mich darüber her. Wochenlang war meine Seele ganz erfüllt von der Kongruenz, der Ähnlichkeit von Dreiecken, Vierecken, Vielecken; ich zergrübelte mein Denken mit der Frage, wo sich eigentlich die Parallelen schneiden; der pythagoräische Lehrsatz bezauberte mich. Dass man seelisch in der Ausbildung rein innerlich ausgebildeter Formen leben könne, ohne Eindrücke der äußeren Sinne, das gereichte mir zur höchsten Befriedigung ... rein im Geiste etwas erfassen zu können, das brachte mir ein inneres Glück. Ich weiß, dass ich an der Geometrie das Glück zuerst kennengelernt habe. ${ }^{\text {221 }}$

Die geometrischen Gesetze sind ein tätig hervorzubringendes Wissen, das ganz von der subjektiven (Denk-) Tätigkeit des Menschen abhängt, gleichzeitig aber in seiner unabhängigen Gesetzlichkeit erlebt wird. Sie empfand Rudolf Steiner als ein Vorbild, wie Geistwissen im Menschen leben sollte:

„Als ein Wissen, das scheinbar von den Menschen selbst erzeugt wird, das aber trotzdem eine von ihm ganz unabhängige Bedeutung hat, erschien mir die Geometrie. Ich sagte mir als Kind natürlich nicht deutlich, aber ich fühlte, so wie die Geometrie muss man das Wissen von der geistigen Welt in sich tragen... Bei der Geometrie sagte ich mir, hier darf man etwas wissen, was nur die Seele selbst durch ihre eigene Kraft erlebt; in diesem Gefühl fand ich die Rechtfertigung, von der geistigen Welt, die ich erlebte, ebenso zu sprechen, wie von der sinnlichen. ${ }^{‘ 22}$

20 Steiner, R.: Mein Lebensgang, GA 28, S. 80f.. Dornach 1962

21 a.a.O., Seite 21

22 a.a.O., Seite $21 \mathrm{f}$. 
Mit diesem Ideal wurde dem Knaben die Natur noch rätselvoller, deren geistigen Hintergrund er ja seit dem geschilderten Ereignis erlebte. Die damals rein materialistisch orientierten naturwissenschaftlichen Begriffe erwiesen sich als ungeeignet, diesen Hintergrund nach dem Vorbild der Geometrie in die Naturerkenntnis einzubeziehen und zu erhellen. So ergab sich dem Dreizehnjährigen die Aufgabe, das Denken so zu gestalten, dass dies möglich werde:

„Ich sagte mir, man kann doch nur zurechtkommen mit dem Erleben der geistigen Welt durch die Seele, wenn das Denken in sich zu einer Gestaltung kommt, die an das Wesen der Naturerscheinungen heran gelangen kann. Mit diesen Gefühlen lebte ich mich durch die dritte und vierte Realschulklasse durch. Ich ordnete alles, was ich lernte, selbst daraufhin an, mich dem gekennzeichneten Ziele zu nähern. ${ }^{، 23}$

Drei Jahre später kann er seine Frage schon konturierter formulieren:

„Auf der einen Seite beschäftigte mich unaufhörlich die Tragweite der menschlichen Gedankenfähigkeit. Ich empfand, dass das Denken zu einer Kraft ausgebildet werden könne, die die Dinge und Vorgänge der Welt wirklich in sich fasst. Ein Stoff, der außerhalb des Denkens liegen bleibt, über den bloß 'nachgedacht' wird, war mir ein unerträglicher Gedanke. Was in den Dingen ist, das muss in die Gedanken des Menschen herein, das sagte ich mir immer wieder.“24

Wenige Jahre später wird Rudolf Steiner an seinen Goethe-Studien klar, „...wie meine Gedanken zu einem Anschauen vom Wesen der Erkenntnis führen, das in Goethes Schaffen und in seiner Stellung zur Welt überall hervortritt. Ich fand, dass meine Gesichtspunkte mir eine Erkenntnistheorie ergaben, die die der Goetheschen Weltanschauung ist. “25

Aus diesen Empfindungen heraus schrieb er die „, Grundlinien einer Erkenntnistheorie der Goetheschen Weltanschauung " zugleich als eine philosophische Begründung seines eigenen geistgemäßen Naturerkennens ${ }^{26}$ und fasst rückblickend zusammen:

23 a.a.O., Seite $37 \mathrm{f}$.

24 a.a.O., Seite 40

25 Siehe Anm. 6, S. 10.

26 Dem Kerngedanken nach hatte Rudolf Steiner seine Sicht bereits vor seiner Auseinandersetzung mit Goethe in einem Aufsatz über den ,Atomismus' formuliert. Diesen Aufsatz schickte er damals Friedrich Theodor Vischer, wie er in seiner Vorrede von 1923 in den ,Grundlinien' schrieb und erhielt zu seiner großen Freude ,einige zustimmende Worte'. „Erst wenn man einsieht, dass es Begriff und Idee ist, was die Wahrnehmung bietet, aber in wesentlich anderer Form als in der von allem empirischen Gehalt befreiten des reinen Denkens, und dass diese Form das Ausschlaggebende ist, begreift man, dass man den Weg der Erfahrung einschlagen muss. Nimmt man aber an, es sei der Inhalt das Maßgebende, dann kann der Behauptung, dass derselbe Inhalt doch auch auf eine von aller Er- 
„Den Geist in der Natur zu finden, sah ich damals nicht als eine Sache des logischen Schließens, oder der Fortsetzung des sinnlichen Wahrnehmens an, sondern als etwas, das sich ergibt, wenn der Mensch vom Wahrnehmen zum Erleben des sinnlichkeitsfreien Denkens sich fortentwickelt ... Wer dem Denken seine über die Sinnesauffassung hinausgehende Wahrnehmungsfähigkeit zuerkennt, der muss ihm notgedrungen auch Objekte zuerkennen, die über die bloße sinnenfällige Wirklichkeit hinaus liegen. Diese Objekte des Denkens sind aber die Ideen. Indem das Denken sich der Idee bemächtigt, verschmilzt es mit dem Urgrund des Weltendaseins; das, was außen wirkt, tritt in den Geist des Menschen ein: er wird mit der objektiven Wirklichkeit auf ihrer höchsten Potenz eins. Das Gewahrwerden der Idee in der Wirklichkeit ist die wahre Kommunion des Menschen. Das Denken hat der Idee gegenüber dieselbe Bedeutung wie das Auge dem Licht, das Ohr dem Ton gegenüber. Es ist Organ der Auffassung. “27

In den folgenden Jahren gelang es Rudolf Steiner, seine naturwissenschaftliche Erkenntnisweise, die er bei Goethe schon anfänglich ausgebildet fand, auf das geistige Wesen des Menschen zu erweitern und in seiner Dissertation über „,Wahrheit und Wissenschaft" eine Menschenkunde der Freiheit zu entwerfen. Aber Eines ist es, selbst zu einem Erkenntniserleben zu kommen, ein Anderes, es mitzuteilen, ohne dass Wesentliches verlorengeht. Eine Darstellungsweise, die dies leisten kann, gelang Rudolf Steiner erstmalig mit seiner „Philosophie der Freiheit". Rückblickend schrieb er:

„Während ich an meiner Philosophie der Freiheit arbeitete, war meine stete Sorge, in der Darstellung meiner Gedanken das innere Erleben bis in diese Gedanken hinein voll wach zu halten. Das gibt den Gedanken den Charakter des mystischen Schauens, macht aber dieses Schauen auch gleich dem äußeren sinnenfälligen Anschauen der Welt. Dringt man zu einem solchen inneren Erleben vor, so empfindet man keinen Gegensatz mehr zwischen Natur- und Geist-Erkennen. Man wird sich klar darüber, dass das zweite nur die metamorphosierte Fortsetzung des ersten ist.“28

Sowohl den naturwissenschaftlichen Arbeiten Goethes wie den geisteswissenschaftlichen Darstellungen Steiners liegt das zum Anschauungsorgan entwickelte Denken zugrunde. Die späteren Darstellungen übersinnlicher Erfahrungen sind stets so gestaltet, dass die Entwicklung des anschauenden Denkens mit ihrer Hilfe möglich wird. Die hierfür erforderliche Darstellungsweise beschrieb Rudolf Steiner einmal folgendermaßen:

„Heute sind die Menschen zufrieden, wenn in einer noch so beliebigen Form dasjenige ausgedrückt wird, was sie sagen wollen. Und wie viele Menschen haben einen

fahrung unabhängige Weise erworben werden könne, nichts entgegengesetzt werden.“ Siehe Anm. 6

27 Steiner, R.: Mein Lebensgang, GA 28, Seite 164f., Dornach 1962.

28 a.a.O., Seite 178 
Begriff davon, was auf geisteswissenschaftlichem Gebiet unbedingt notwendig ist, dass sprachkünstlerischer Sinn für eine jegliche Darstellung nötig wäre. ‘29

Daher leuchtet es ein, wenn Steiner in einer Vorrede zur „Theosophie“ schreibt:

„Wie man Bücher in unserem Zeitalter zu lesen pflegt, kann dieses nicht gelesen werden. In einer gewissen Beziehung wird von dem Leser jede Seite, ja mancher Satz erarbeitet werden müssen. Das ist mit Bewusstsein angestrebt worden. Denn nur so kann das Buch dem Leser werden, was es ihm werden soll. Wer es bloß durchliest, der wird es gar nicht gelesen haben. Seine Wahrheiten müssen erlebt werden. Geisteswissenschaft hat nur in diesem Sinne einen Wert. “30

Schmerzlich musste er jedoch in den folgenden Jahren erleben, dass seine Anregungen zu wenig aufgegriffen wurden:

„Und das ist dasjenige, was innerhalb der anthroposophischen Bewegung erlebt werden sollte: diese Notwendigkeit, die Gedanken, die man zunächst als Gedanken empfängt, als Lebensmächte zu empfinden. Ich werde jetzt etwas ganz Paradoxes sagen: Manchmal begreift man die Gegner der Anthroposophie viel besser als die Anhänger. Die Gegner sagen: Ach, diese anthroposophischen Gedanken sind phantastisch, sie entsprechen keiner Wirklichkeit. - Die Gegner weisen sie ab, sie sind nicht weiter von ihnen berührt. Man kann ein solches Verhältnis gut verstehen, man kann die verschiedensten Gründe dafür anführen, meistens ist es die Furcht vor diesen Gedanken, die nur unbewusst bleibt, aber immerhin, es ist ein Verhältnis. Oftmals aber kommt dieses vor, dass die Gedanken zwar aufgenommen werden, dass man aber durch die Gedanken, die von alledem abweichen, was sonst in der Welt aufgenommen werden kann, nicht einmal so viel fühlt, wie man fühlt, wenn man an den Knopf einer Elektrisiermaschine den Knöchel hält und elektrisiert wird. Da fühlt man durch den elektrischen Funken wenigstens körperlich einiges Zucken. Ein solches Einschlagen eines Funkens in die Seele ist dasjenige, was einem, wenn es nicht vorhanden ist, so ungeheuren Schmerz machen kann. Dies hängt mit dem zusammen, dass unsere Zeit notwendig hat für die Menschen, nicht nur vom Physischen ergriffen zu werden, sondern notwendig hat, vom Geistigen ergriffen und gepackt zu werden. Der Mensch vermeidet es, gestoßen, gezerrt zu werden, aber er vermeidet es nicht, Gedanken an sich herankommen zu lassen, die von andern Welten handeln, die sich als etwas ganz Besonderes in die gegenwärtige Welt der Sinne hereinstellen, und vermeidet es nicht, diesen Gedanken gegenüber dieselbe Gleichgültigkeit zu haben wie den Gedanken der Sinne gegenüber.“31

Denjenigen, die geisteswissenschaftliche Schriften so lesen wollen wie ein gewöhnliches Buch, wie ein Rechen-, Chemie- oder Botanikbuch ${ }^{32}$ oder gar einen

29 Steiner, R., GA 59, S. 38 ff. (20. Januar 1910). Siehe auch GA 119, S. 102f. und GA 127, S. $82 \mathrm{f}$.

30 Steiner, R.: Theosophie, GA 9, Seite 12. Dornach 2003

31 GA 223, Seite 116ff.

32 GA 169, S. 43 f. 
Sensationsroman $^{33}$ empfahl er in den zwei Jahrzehnten nach Erscheinen der "Theosophie “ immer wieder, statt anthroposophischer Literatur lieber Kochbücher $^{34}$ oder eine Abhandlung über Tarifverträge ${ }^{35} \mathrm{zu}$ lesen, weil das nützlicher wäre. Seine Ausführungen zu den besonderen Anforderungen des Studiums der Anthroposophie werden in diesen zwanzig Jahren zahlreicher, ausführlicher, eindringlicher und deutlicher. ${ }^{36}$ So stellte er 1923 in einem Vortrag vor Mitgliedern der Anthroposophischen Gesellschaft fest:

\begin{abstract}
„Anthroposoph werden heißt eben nicht bloß, Anthroposophie als Theorie kennenlernen, sondern Anthroposoph sein erfordert in einem gewissen Sinne eine Seelenumartung. Diese aber wollen gewisse Menschen nicht. Daher wurde es auch nie verstanden, wenn ich gesagt habe: Es gibt zweierlei Arten, sich zum Beispiel mit meinem Buche Theosophie zu beschäftigen. Die eine ist, es zu lesen oder meinetwillen, es auch zu studieren, indem man mit der gewöhnlichen Seelenverfassung an es herangeht und es im Sinne dieser gewöhnlichen Seelenverfassung beurteilt. Dann ist der Seelenvorgang der Qualität nach ganz der gleiche, ob man eine Theosophie liest oder ein Kochbuch. Für den Wert des Erlebens ist dann kein Unterschied zwischen dem Lesen dieser Theosophie und dem Lesen eines Kochbuches, nur dass man, wenn man das tut, eben einfach im Lesen der Theosophie auf einer höheren Stufe träumt, nicht lebt. ${ }^{\text {‘37 }}$
\end{abstract}

Wie kann an der Verwandlung des Denkens zu einem bewussten Wahrnehmungsorgan gearbeitet werden ? Dazu sollen im Folgenden einige Anregungen gegeben werden. ${ }^{38}$

33 GA 229, S. 13

34 GA 119, S.102f., GA 169, S. 43f., GA 182, S. 71, GA 196, S.190f., GA 257, S. 131 und $180 \mathrm{f}$.

35 GA 196, S. $190 \mathrm{f}$.

36 Die folgende nicht systematische Stellensammlung wurde in Zusammenarbeit mit Prof. Tomas Zdrazil (Freie Hochschule Stuttgart) erstellt: 1886: GA 2, S. 30, 1904: GA 9, S. 12, GA 10, S. 52, GA 89, S. 91, 1907: GA 99, S. 158ff., GA 56, S. 208, a. a.O., S. 224, 1910: GA 13, S. 29, a. a.O., S. 49-51, GA 119, S. 102f., 1911: GA 14, S. 161, GA 127, S. 82f., 1912: GA 16, S. 9, 1914: GA 156, S. 77, 1916: GA 169, S. 43f., 1917: GA 176, S. 116, 1918: GA 71, S. 182, GA 181, S. 425, 1920: GA 196, S. 190f., GA 301, S. 62 ff., a. a.O., S.115, GA 334, S. 244, GA 322, S. 111, 1922: GA 212, S. 158, GA 344, S. 187 f., GA 217, S. 125f., 1923: GA 221, S. 38, a. a. O., S. 45ff., GA 257, S. 131, a. a. O., S. 180f., GA 84, S.19f., GA 223, S. 116ff., a .a. O., S. 155, GA 229, S. 13, 1925: GA 28, S. 435

37 GA 257, S. 131

38 Siehe auch: Teichmann, F.: Auferstehung im Denken. Stuttgart 1996. Schmidt, R.: Wie lässt sich Reinkarnation denken? Dornach 2010. Kühlewind, G.: Die Wahrheit tun, Kap.: Das Studium, Stuttgart 1982. Kühlewind, G.: Vom Normalen zum Gesunden, Kap: Das Studium, Stuttgart 1995. Lindenau, Chr.: Der übende Mensch, Stuttgart 2001. Smit, J. u. a.: Freiheit erüben. Stuttgart 1988 u. a. m. 


\title{
Anregungen zur seminaristischen Arbeit
}

Zur Einstimmung und um die eigene Anschauungskraft zu wecken, nehmen wir irgendeinen Gegenstand aus der Natur, einen Apfel etwa oder auch ein abstraktes Gemälde ist möglich (und von den Teilnehmern besser einsehbar). Nun versuchen wir eine Viertelstunde etwa so konzentriert und aufmerksam wie möglich nur zu beschreiben, was an dem Gegenstand wahrnehmbar ist. Diese anschauende und beschreibende Tätigkeit fällt uns - auch wenn sie ungewohnt sein mag - beim äußeren Sehen leichter, als an einer begrifflichen Darstellung und mancher Teilnehmer bemerkt dabei, dass er einen Apfel noch nie wirklich gesehen hat.

Anschließend wenden wir die am Sinnlichen geweckte anschauende Kraft der Ideengestaltung einer geisteswissenschaftlichen Darstellung zu, indem wir die Gedanken des Textes ebenso beschreiben, wie vorher den Naturgegenstand oder das Bild. Gleich im Anfang ergibt sich eine besondere Chance seminaristischer Arbeit: die Korrektur. Bei der sinnlichen Wahrnehmung wird ein Irrtum zumeist sehr schnell durch die Wirklichkeit korrigiert. Beim Auffassen von Gedanken tritt diese Korrektur nicht ohne Weiteres ein, so dass man beim ersten oder sogar mehrmaligen Lesen gar nicht den ,Gegenstand' vor Augen hat, sondern seinen irrtümlichen Gedanken. Anders beim skizzierten gemeinsamen Studium: Versucht ein Teilnehmer die Gedanken des Textes in eigenen Worten zu beschreiben, können die anderen unschwer unterscheiden, ob es sich dabei um die vorliegenden Gedanken handelt oder um Assoziationen, Folgerungen, Interpretationen oder gar Phantasieprodukte. Es geht bei diesem ersten Üben gedanklicher Wahrnehmung weder um das

\begin{abstract}
„....gläubige Annehmen, noch um das ungläubige Ablehnen ... Der Zuhörer (bzw. Leser, d. V.) soll die Gedanken, die ihm mitgeteilt werden nur nachdenken, frei von Glauben und Zweifel, frei von Ja und Nein. Er muss sich ,neutral' einstellen und ,probeweise' die Lehre im Geiste wirken lassen ... Der Zuhörer soll ohne ausgesprochenes Ja oder Nein einen Gedanken wie etwas ganz Objektives hinnehmen. ${ }^{\text {339 }}$
\end{abstract}

So kann, wie bei dem in der Vorübung gegebenen äußeren Bild, ein inneres Gedankenbild, eine Ideenlandschaft entstehen, deren Motive und Linien gemeinsam immer klarer und deutlicher herausgearbeitet werden können.

Auf diese Weise ergibt sich beim Studium eines geisteswissenschaftlichen Buches ein inneres Mitproduzieren:

„Wenn Sie geisteswissenschaftliche Bücher lesen, so hat das gar keinen Wert, wenn

Sie innerlich nicht nachkonstruieren. Dann ist es ein inneres Mitproduzieren. Wenn das nicht der Fall ist, dann ist es kein Studium geisteswissenschaftlicher Bücher.“ ${ }^{\text {40 }}$

39 GA 89, S. 91, 26. Mai 1904

40 GA 56, S. 208, 3. Dez. 1907 
Für dieses an die exakte Anschauung sich haltende Üben, sind geisteswissenschaftliche Bücher geschrieben:

„Es muss so geschrieben sein, dass es die Eigentätigkeit hervorruft. Je mehr man sich selbst abplagen muss, je mehr zwischen den Zeilen steht, desto gesünder ist es. “41

„Die ruhige, besonnene Gedankenanstrengung, die dieses Lesen notwendig macht, verstärkt die Seelenkräfte und macht sie dadurch fähig, der geistigen Welt nahe zu kommen. ${ }^{442}$

An der Ideenlandschaft einer geisteswissenschaftlichen Darstellung kann sich auf diese Weise eine Art Tasterlebnis, ein Gegenstandsbewusstsein bilden, wie wir es sonst aus der äußeren Sinneswelt kennen.

Ein tieferes Verständnis einer geisteswissenschaftlichen Darstellung kann sich mit der Frage nach dem Zusammenhang der Einzelmotive in der entstandenen Ideenlandschaft ergeben. Als leitendes Vorbild kann hierbei das mathematische Denken bzw. geometrisches Beweisen dienen, wie es Rudolf Steiner bereits in jungen Jahren als Ideal geisteswissenschaftlichen Wissens erfasst hatte (siehe oben).

Nehmen wir zum Beispiel die Behauptung, dass in der ebenen Geometrie die Summe der Innenwinkel eines Dreiecke stets den einer Geraden, d.h. einen gestreckten Winkel ergeben (Winkelsumme $=180^{\circ}$ ). Um uns davon zu überzeugen stellen wir uns ein beliebiges Dreieck vor. Jetzt ziehen wir durch eine frei wählbare Ecke des Dreiecks eine Parallele zur gegenüberliegenden Seite. Verlängern wir nun die beiden übrigen Seiten über die gewählte Ecke hinaus, so können wir ,sehen', dass alle drei Innenwinkel in der gewählten Ecke sich wiederholen und gemeinsam $180^{\circ}$ ergeben, womit unsere Eingangsbehauptung bewiesen ist. Der bewiesenen Zusammenhang lässt sich für uns jetzt gleichsam am Dreieck , ablesen', er ist ,ersichtlich' geworden.

Ebenso kann der Zusammenhang vom ersten und zweiten Absatz der „Theosophie “ in dieser Art ,sichtbar' werden. ${ }^{43}$ Im zweiten Absatz wird die eigene Aufmerksamkeit, wie sie sich im Goethe'schen Eingangsgedanken betätigte zum Gegenstand der Beobachtung. Ebenso wie bei Goethe handelt es sich auch im zweiten Schritt Rudolf Steiners um eine innere Beobachtung, aber eine solche, die ganz auf den Zusammenhang gerichtet ist, sich ganz im Geistigen, in gedanklicher Beobachtung abspielt. Diese Beobachtung an der eigenen Aufmerksamkeit kann jeder Mensch machen. Der Zusammenhang der beiden ersten Motive der „Theosophie“ erscheint in der Welt (am Subjekt) auf vergleichbare

41 a. a. O., S.224, 5. Dez.1907

42 GA 13, S. 29

43 GA 9, Kapitel: Das Wesen des Menschen 
Weise wie das Gesetz über die Winkelsumme eines ebenen Dreiecks. Er ist auch ohne mein besonderes Subjekt vorhanden, gehört der Welt an.

Eine weitere Stufe geisteswissenschaftlichen Erlebens wird möglich, wenn wir beginnen auf die Art der Darstellung zu achten, z.B. in den Betrachtungsarten, wie sie zur Charakterisierung der leiblichen und der geistigen Wesenheit des Menschen in der „Theosophie“ unterschieden werden. Es genügt, jeweils die drei ersten Sätze zu vergleichen, um zu bemerken, wie der Leib als Gegebenes, Vorhandenes geschildert wird, während die geistige Wesenheit des Menschen eigenständig erst mal gar nicht vorhanden, sich zunächst zart in der Seele ankündigt, in der Seele Folgen zeitigt und sich dann erst - mit dem dritten Satz - in einer Betätigung zu erkennen gibt.

Will man die innere Dramatik einer geisteswissenschaftlichen Darstellung erschließen, muss man auf deren Komposition achten, sie wie eine Partitur lesen lernen ${ }^{44}$ :

„Versuchen Sie einmal, wirkliche Darstellungen der geisteswissenschaftlichen Materie zu prüfen. Da werden Sie finden, dass derjenige, der als wahrer, echter Geisteswissenschafter solche Dinge geschrieben hat, auch wirklich daran gearbeitet hat, um künstlerisch einen jeden Satz auszugestalten, und dass da nicht in beliebiger Weise ein Zeitwort am Ende oder am Anfang steht. Da werden Sie finden, dass ein jeder solcher Satz eine Geburt ist, weil er innerlich seelisch, nicht bloß als Gedanke, sondern als unmittelbare Form erlebt werden soll. Und wenn Sie den Zusammenhang des Dargestellten verfolgen, dann werden Sie sehen, dass bei drei aufeinanderfolgenden Sätzen, der mittlere nicht bloß an den ersten angehängt ist und der dritte wiederum an den vorhergehenden, sondern Sie werden finden, dass derjenige, der Geisteswissenschaftliches darstellt, nicht bloß den ersten Satz, sondern auch den dritten Satz bereits fertig in seiner Anlage hat, bevor er den mittleren gestaltet, weil die Wirkung des mittleren Satzes von dem abhängen soll, was als Wirkung des ersten Satzes zurückbleibt und wiederum auf den nächstfolgenden Satz übergehen kann. “45

Eine Partitur muss man horizontal und vertikal vor sich haben: ,horizontal' die verschiedenen Instrumente - entsprechend den einzelnen inhaltlichen Motiven einer Darstellung - und ,vertikal', indem man diese vorauserlebend den richtigen Einsatz zu geben vermag. Die Komposition erklingt innerlich, auch ohne dass sie äußerlich in Erscheinung zu treten braucht. Dabei bewegt man sich in einem sinnlichkeitsfreien Denken, das zugleich ein Erleben ist und das aus einer frei ergriffenen Willensbetätigung hervorgeht, man lebt im reinen Denken.

„Man lebt mit dem reinen Denken im Übersinnlichen: aber man erlebt nur dieses auf eine übersinnliche Art; man erlebt noch nichts anderes Übersinnliches. Und das

44 GA 301, Seite 115, GA 334, Seite 244, GA 322, Seite 111

45 Siehe Anm. 29 
übersinnliche Erleben muss sein eine Fortsetzung desjenigen Seelen-Erlebens, das schon im Vereinigen mit dem reinen Denken erreicht werden kann. Deshalb ist es so bedeutungsvoll, diese Vereinigung richtig erfahren zu können. Denn von dem Verständnisse dieser Vereinigung aus leuchtet das Licht, das auch rechte Einsicht in das Wesen der übersinnlichen Erkenntnis bringen kann. ${ }^{\text {“46 }}$ 